\title{
The Effectiveness of a Liver Disease Education Class for Providing Information to Patients and Their Families
}

\author{
Yukio Kadokawa ${ }^{\mathrm{a}}$, Kazuhiro Katayama ${ }^{\mathrm{b}, \mathrm{e}}$, Kozo Takahashia ${ }^{\mathrm{a}}$, Nobuhisa Fukushima ${ }^{\mathrm{a}}$, Setsu Tanaka ${ }^{\mathrm{c}}$, \\ Yuko Taniguchid $^{\mathrm{d}}$, Takatoshi Nawa ${ }^{\mathrm{b}}$, Mitsuru Sakakibara ${ }^{\mathrm{b}}$, Kazuyoshi Ohkawa ${ }^{\mathrm{b}}$
}

\begin{abstract}
Background: We have been conducting liver disease education classes regularly in our hospital for the purpose of providing health information to patients and their families.

Methods: In order to evaluate the effectiveness of these classes, we conducted a questionnaire survey of patients and family members who attended the classes held three times in 2012. The cumulative total number of participants was 80 (49 patients, 26 family members, and five others). The classes focused on the following areas: 1) prevention of hepatic cancer; 2) treatment of hepatic cancer; 3) iron restriction diet for hepatitis $\mathrm{C}$ patients; and 4) importance of branchedchain amino acid preparations. Self-evaluation of knowledge in these areas was based on a four-point scale.
\end{abstract}

Results: A comparison of knowledge levels between the patients and their family members revealed no statistically significant differences. Therefore, subsequent analyses were performed by combining the patients and their families into one group. The knowledge level of the participants increased with the number of class attendances; that is, the more often they attended, the more they accumulated knowledge (Kruskal-Wallis test: $\mathrm{P}<0.0001 ; \mathrm{P}=0.0368 ; \mathrm{P}=$ 0.0021 ; and $\mathrm{P}<0.0001)$. In addition, the results of the questionnaire administered immediately before and after the education class showed significant improvement in the knowledge level for each area.

Conclusion: The results of this study indicate the liver disease education class to be effective for improving the knowledge of patients and

Manuscript accepted for publication January 12, 2017

aDepartment of Pharmaceutical Services, Osaka Medical Center for Cancer and Cardiovascular Diseases, Osaka, Japan

${ }^{b}$ Department of Hepatobiliary and Pancreatic Oncology, Osaka Medical Center for Cancer and Cardiovascular Diseases, Osaka, Japan

'Department of Nursing, Osaka Medical Center for Cancer and Cardiovascular Diseases, Osaka, Japan

${ }^{\mathrm{d} D e p a r t m e n t ~ o f ~ N u t r i t i o n, ~ O s a k a ~ M e d i c a l ~ C e n t e r ~ f o r ~ C a n c e r ~ a n d ~ C a r d i o v a s c u-~}$ lar Diseases, Osaka, Japan

${ }^{\mathrm{e} C}$ Corresponding Author: Kazuhiro Katayama, Department of Hepatobiliary and Pancreatic Oncology, Osaka Medical Center for Cancer and Cardiovascular Diseases, 1-3-3, Nakamichi, Higashinari-ku, Osaka, 537-8511, Japan. Email:katayama-ka@mc.pref.osaka.jp

doi: https://doi.org/10.14740/jocmr2914w their families. The importance of repeated information provision was also demonstrated.

Keywords: Repeated education; Knowledge level; Cancer; Nutrition; Branched-chain amino acid

\section{Introduction}

Chronic liver diseases require long-term management. Patient understanding of these diseases may contribute to controlling the course and the symptoms of liver diseases. However, chronic liver disease can be extensive and encompasses a wide variety of conditions including chronic hepatitis, cirrhosis, and hepatic cancer. Therefore, patients are required to master a broad range of knowledge to manage these diseases. Often, patients must make difficult decisions about their own treatment. However, it is not easy for physicians to provide sufficient information for patients during routine appointments of out-patients department because the amount of time that can be spent with one patient is quite limited. Nagao et al pointed out that providing the latest and most accurate information is important for the treatment decision-making process in patients with chronic hepatitis [1].

Studies have shown treatment coupled with a patient education program to be more effective than conventional treatment for improving both quality of life (QOL) and disease control in patients with chronic diseases. Roumie et al reported that a patient education program, designed to provide information, effectively improved the control of blood pressure in patients with hypertension [2]. Bourbeau et al examined the effectiveness of a self-management education program for patients with chronic obstructive pulmonary disease [3]. They reported that this treatment coupled with a self-management education program was more effective for improving disease control than conventional treatment. In addition, there are studies showing the effectiveness of an intervention program focusing on information provision for improving the psychological health of patients with diabetes mellitus, chronic heart failure, and other chronic diseases [4-7]. Thus, the effects of patient education programs on disease control and QOL improvement have been established. However, the mechanisms by which such programs exert their beneficial effects have yet to be elucidated.

In Japan, many hospitals are currently conducting liver 
Table 1. Knowledge Degree of Patients and Family and the Times of Experience to Attend Classrooms

\begin{tabular}{|c|c|c|c|}
\hline $\begin{array}{l}\text { Tips of information/times } \\
\text { of experience of classroom }\end{array}$ & Patients & Family & $P$ value \\
\hline \multicolumn{4}{|l|}{ Prophylaxis of $\mathrm{HC}$} \\
\hline 0 & $1.00 \pm 0.82$ & $0.67 \pm 0.49$ & 0.3034 \\
\hline $1-2$ & $1.36 \pm 0.78$ & $1.30 \pm 0.48$ & 0.7027 \\
\hline$\geq 3$ & $2.30 \pm 0.48$ & $1.67 \pm 0.57$ & 0.0958 \\
\hline \multicolumn{4}{|l|}{ Treatment of HC } \\
\hline 0 & $1.33 \pm 1.16$ & $0.75 \pm 0.71$ & 0.3305 \\
\hline $1-2$ & $1.27 \pm 0.96$ & $0.83 \pm 0.41$ & 0.2824 \\
\hline$\geq 3$ & $2.00 \pm 0.63$ & 2.00 & $>0.99$ \\
\hline \multicolumn{4}{|l|}{ Iron restriction } \\
\hline 0 & $1.00 \pm 0.89$ & $0 \pm 0$ & 0.0258 \\
\hline $1-2$ & $1.17 \pm 0.39$ & $1.00 \pm 0$ & 0.4631 \\
\hline$\geq 3$ & $2.75 \pm 0.50$ & $1.50 \pm 0.71$ & 0.0801 \\
\hline \multicolumn{4}{|l|}{ Effects of BCAA } \\
\hline 0 & $0.60 \pm 0.70$ & $0.17 \pm 0.58$ & 0.0521 \\
\hline $1-2$ & $0.85 \pm 0.66$ & $0.56 \pm 0.73$ & 0.2278 \\
\hline$\geq 3$ & $2.30 \pm 0.68$ & $1.33 \pm 0.58$ & 0.0564 \\
\hline
\end{tabular}

Comparisons were performed using Mann-Whitney test. Data were shown as mean \pm SD. HC: hepatic cancer; BCAA: branched-chain amino acid.

disease education classes for the provision of information to patients with chronic liver disease. Although the significance of such education classes has been recognized by patients and health care providers, there are unresolved issues regarding the cost of administration and the method of evaluating their effectiveness [8]. We have been conducting a liver disease education class regularly in our hospital since 2009. We also have been evaluating the effectiveness of this class using a questionnaire with the aim of improving our program. In the present study, we analyzed the results obtained from the questionnaire administered in 2012. Herein, we report the findings on knowledge acquisition by the class participants.

\section{Methods}

\section{Subjects and methods}

A questionnaire was administered to participants in our liver disease education classes attended in 2012 (February, July, and November) at our hospital. The cumulative total number of participants was 80 (49 patients, 26 family members, and five others). They consisted of 40 men and 37 women, while gender was unknown in three, with a mean age (standard deviation (SD)) of 67.1 (12.3) years. The participants were asked to respond to self-evaluation questions pertaining to their knowledge of prevention, treatment, and drugs. The association between the knowledge level and the number of class attendances in the past was analyzed.

\section{Ethical approval and informed consent}

Written informed consent was obtained from the participants for data publication. Our retrospective study was approved by the Institutional Review Board of our hospital (No. 1511109159), and was performed in accordance with the ethical standards as laid down in the 1964 Declaration of Helsinki and its amendments of compatible ethical standard.

\section{Questionnaire on knowledge level}

The liver disease education class in our hospital focuses on the following areas: 1) prevention of hepatic cancer; 2) treatment of hepatic cancer; 3) iron restriction diet for hepatitis C patients; and 4) importance of branched-chain amino acid preparations. The knowledge levels of the participants for each area were self-evaluated semi-quantitatively using a four-point scale. In addition, the questionnaire was administered immediately before and after class attendance in order to evaluate knowledge acquisition. Score 0 means I know nothing whatsoever about this subject, or I did not understand it at all. Score 1 means I have little knowledge of this subject, or I did not understand it well. Score 2 means I know this subject rather well, or I mostly understood it. Score 3 means I know this subject very well, or I understood it thoroughly.

We divided the participants into three groups according to the number of class attendances in the past in order to examine 
(a)

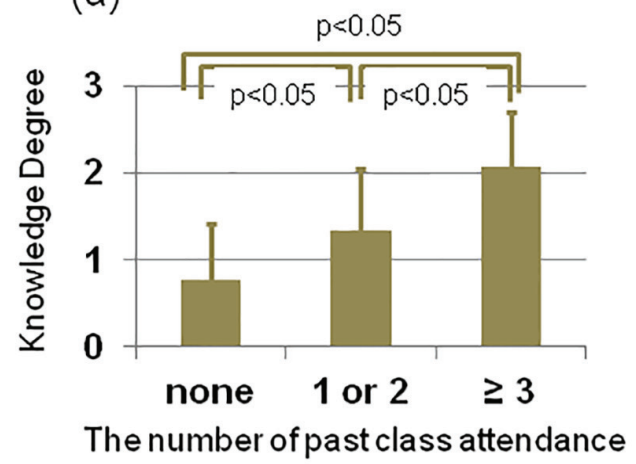

(b)

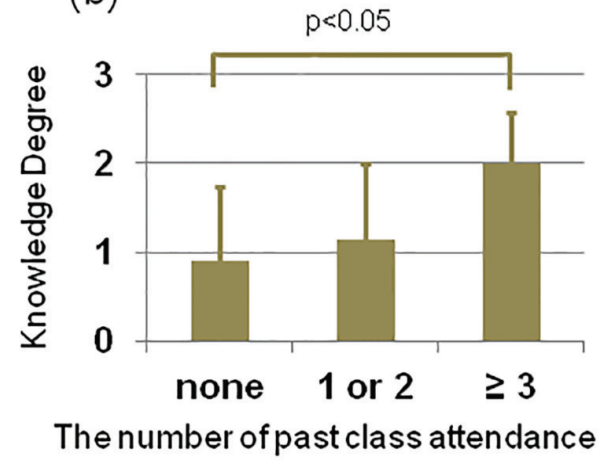

(c)

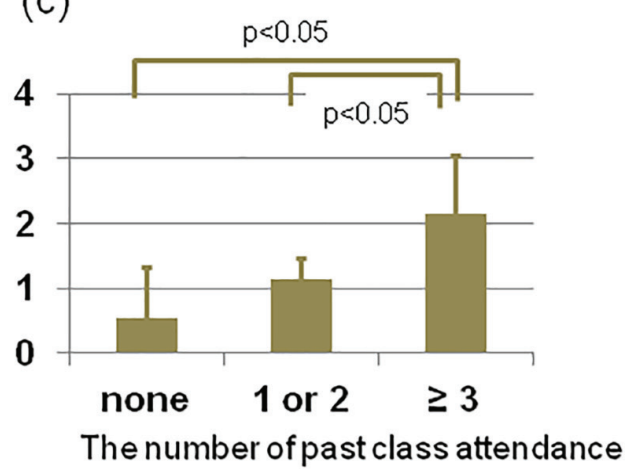

(d)

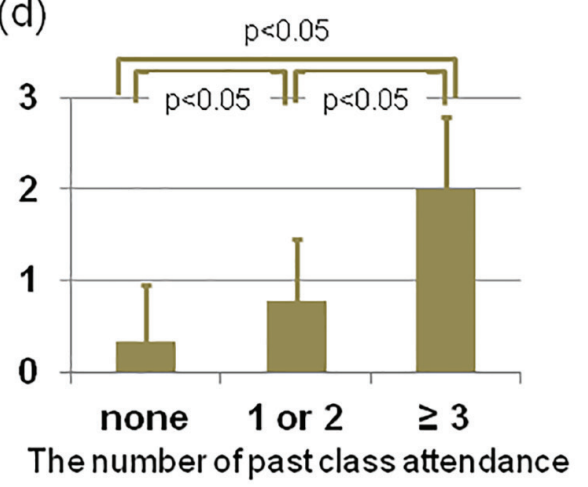

Figure 1. Relationship between knowledge degree just before the lecture and times of attendance to classroom for liver diseases. Because comparison (Kurskal-Wallis) of multi-groups revealed significant difference in $(a)(P<0.0001),(b)(P=0.0388)$, (c) $(P=0.0021)$, and $(d)(P<0.0001)$, Mann-Whitney test was conducted. $P$ values by Mann-Whitney test are indicated. (a) Prophylaxis of hepatic cancer. (b) Treatment of hepatic cancer. (c) Significance of iron restrictions in the C type liver disease. (d) Significance of BCAA.

the association between their knowledge levels and the number of class attendances. 1) Zero times: This is my first time attending the class. 2) One to two times: This is my second or third time attending the class. 3) Three times or more: This is my fourth or greater time attending the class.

Given that the first group (no classes attended in the past) was free from any possible effects of the education class, we assumed that the knowledge levels of this group reflected the level of knowledge acquired through conventional treatment without a patient education program. Using this group as a control, we evaluated the effects (both immediate and longterm effects) of the education class program.

This questionnaire was completely anonymous.

\section{Statistical analysis}

Multiple comparisons of knowledge levels according to the number of education class attendances in the past were performed using a Kruskal-Wallis test with a significance level of $\mathrm{P}<0.05$. When a significant difference was observed, the Mann-Whitney test was additionally applied. Comparisons of knowledge levels between patients and their families were performed using the Mann-Whitney test. Comparisons of knowledge levels between immediately before and after class attend- ance were performed using the Wilcoxon test.

\section{Results}

Comparison of knowledge levels between patients and their family members

A comparison of knowledge levels between the patients and their family members according to the number of liver disease education class attendances in the past revealed no significant difference, although the patients tended to have higher levels of knowledge in each area than their families. Therefore, subsequent analyses were performed by combining the patients and their family members into one group (Table 1).

Relationships between the knowledge level for all items immediately before attending the class and the number of liver disease education class attendances in the past

The relationship between the knowledge level for each item before attending the class and the number of past class attendances is shown in Figure 1. The results indicate that the knowl- 
Table 2. Changes of Knowledge Degree of Participants by Attending Classrooms

\begin{tabular}{|c|c|c|c|}
\hline $\begin{array}{l}\text { Tips of information/times of } \\
\text { experience of classroom }\end{array}$ & Patients & Family & P value \\
\hline \multicolumn{4}{|l|}{ Prophylaxis of HC } \\
\hline 0 & $0.76 \pm 0.63$ & $2.05 \pm 0.38$ & $<0.0001$ \\
\hline $1-2$ & $1.34 \pm 0.71$ & $2.09 \pm 0.45$ & $<0.0001$ \\
\hline$\geq 3$ & $2.07 \pm 0.62$ & $2.54 \pm 0.52$ & 0.0956 \\
\hline \multicolumn{4}{|l|}{ Treatment of $\mathrm{HC}$} \\
\hline 0 & $0.91 \pm 0.83$ & $2.00 \pm 0.0$ & 0.0339 \\
\hline $1-2$ & $1.14 \pm 0.85$ & $1.91 \pm 0.54$ & 0.0114 \\
\hline$\geq 3$ & ND & ND & ND \\
\hline \multicolumn{4}{|l|}{ Iron restriction } \\
\hline 0 & $0.54 \pm 0.78$ & $2.31 \pm 0.63$ & 0.0012 \\
\hline $1-2$ & $1.13 \pm 0.35$ & $2.54 \pm 0.52$ & 0.0017 \\
\hline$\geq 3$ & $2.14 \pm 0.90$ & $3.00 \pm 0.0$ & 0.0633 \\
\hline \multicolumn{4}{|l|}{ Effects of BCAA } \\
\hline 0 & $0.32 \pm 0.63$ & $1.86 \pm 0.56$ & $<0.0001$ \\
\hline $1-2$ & $0.78 \pm 0.68$ & $1.85 \pm 0.71$ & $<0.0001$ \\
\hline$\geq 3$ & $2.00 \pm 0.78$ & $2.46 \pm 0.69$ & 0.0588 \\
\hline
\end{tabular}

Comparisons were performed using Wilcoxon test. Data were shown as mean \pm SD. HC: hepatic cancer; BCAA: branched-chain amino acid; ND: not determined because small number of subjects.

edge level for each item improved significantly as the number of class attendances increased. The participants who achieved a mean score of 2.0 (the "mostly understood" knowledge level) or more on every item had all attended the class at least three times in the past.

\section{Changes in the knowledge level for items after attending the class}

The changes in the knowledge level for each of the items according to the number of education class attendances in the past are shown in Table 2. Those who had attended the education class zero to two times in the past showed a significant improvement in their knowledge level. On the other hand, those who had attended the education class three times or more showed only a tendency for improvement, which did not reach statistical significance.

\section{Discussion}

There was no significant difference in knowledge levels between the patients and their family members, although the knowledge level of the patients tended to be higher than that of their families. It should be noted that the family members in the present study were those who attended the education classes with the patients, implying that they were highly motivated to learn about liver diseases. Therefore, we conducted subsequent analyses by combining the patients and their fam- ily members into one group.

This study yielded two important findings. First, the knowledge level of the patients and their family members who had not participated in the education class in the past was not satisfactory. In particular, the mean score for the knowledge level pertaining to the prevention of liver cancer was 1 (little knowledge of this subject, did not understand it well), despite such knowledge being important for all participants. Since all patients and family members were supposed to have been provided basic health information by physicians, the findings of this study suggest limitations in the provision of such information in routine clinical practice. One possible explanation would be that patients had difficulty remembering the information provided by their physicians during their regular consultations. Another possible explanation would be that the information provided by physicians might be insufficient. In our view, these two explanations are not mutually exclusive. It is conceivable that the information provision in routine clinical practice tends to focus on the current, perhaps ongoing, treatment regimen, as well as alternative treatment plans that might be implemented in the near future, rather than the issues pertaining to long-term care. This may partly be attributable to the limited time allotted for consultation in routine clinical practice. Under such circumstances, patients and their family members may have difficulty remembering or ascertaining the importance of the information provided to them. There is also a possibility that patients lack sufficient motivation to commit to memory and/or act on what they have learned. It is unlikely that physicians had not provided information about the prevention of liver cancer since this is one of the major objectives in 
the management of chronic liver diseases. Given that patients with chronic liver disease often have few subjective symptoms, the exception being those in advanced disease states, some may lack motivation due to not fully perceiving the longterm threats to their health and QOL. Further investigations are needed to clarify these issues.

Second, the knowledge levels of the patients and their family members improved with class attendance and the degree of these improvements differed according to the number of class attendances. This suggested systematic information provision to be effective for improving knowledge levels and that attending a certain number of classes would be required to achieve a satisfactory outcome. In the present study, all of those who achieved a score of at least 2.0 (the "mostly understood" knowledge level) had participated in the education class program at least three times in the past, suggesting repeated information provision to be important for knowledge acquisition.

It should be noted that, while the knowledge level immediately before class attendance increased with the number of education class attendances, the knowledge levels immediately after the class were high even for those with fewer class attendances in the past (Table 2). This was probably related to the effect of the class being most evident immediately after attendance, although there was a possibility that the knowledge obtained by those who had attended the education class in the past was more robust compared with that of those who had not. This notion was supported by the finding that the patients who had attended the education class at least three times in the past showed a mean score immediately before the class of about 2.0 , indicating that their knowledge level had been mostly retained since the prior class participation. On the other hand, the patients who had attended the education class only once or twice in the past showed a mean knowledge level before the class of about 1.0, indicating that their knowledge level at the time of the prior participation (about 2.0) had not been retained. Based on these results, we consider repeated information provision (at least three times) to be important for maintaining an adequate knowledge level.

Health literacy is a concept that has received attention in recent years. Health literacy encompasses peoples' knowledge, motivation, and competencies to obtain, understand, evaluate, and apply health information in order to make judgments and decisions concerning healthcare, disease prevention, and health promotion, with the goals of maintaining or improving QOL over the course of their lives [9]. Modern society is characterized by remarkable diversification and people are constantly inundated with information. It is difficult to keep track of all the information currently available. There is also major diversification of individual values, prompting people to seek more individualized information. Therefore, individuals need to have the ability to search for and obtain information that is beneficial to them. Sophie Bostock and her colleagues reported that one-third of older adults in England did not have sufficient health literacy; that is, they had difficulty in reading and understanding basic health-related written information and that poorer understanding was associated with mortality [10]. Thus, improving health literacy can reasonably be expected to contribute to the control of liver disease.
The major limitation of this study involves the method used for assessing knowledge levels. The self-evaluation method was employed and there was no objective measurement. However, we believe the self-evaluation method used in this study to be valid because the results correlated closely with the number of education class attendances. Further studies investigating the relationships between knowledge levels and aspects of treatment efficiency, including compliance with oral drugs, are needed.

\section{Conclusion}

The present results confirm our liver disease education class to be effective for improving the knowledge levels of participants. It was also demonstrated that repeatedly attending education classes contributed to maintenance of the knowledge acquired by participants. We can reasonably anticipate that improving the knowledge level of patients would enhance compliance with drug treatments and reduce anxiety in patients with liver disease. Patient education programs merit further study.

\section{Acknowledgments}

The authors would like to thank all medical stuffs in our education team for date collection.

\section{Conflicts of Interest}

K. Katayama has received a speaker honorarium from Ajinomoto Pharmaceuticals Co., Ltd. The other authors declare that they have no conflicts of interest.

\section{Funding}

This research was partly supported by a grant-in-aid from Seijinbyo-Yobou Kyokai.

\section{References}

1. Nagao Y, Kawakami Y, Yoshiyama T, Sata M. Analysis of factors interfering with the acceptance of interferon therapy by HCV-infected patients. Med Sci Monit. 2008;14(11):PI45-52.

2. Roumie CL, Elasy TA, Greevy R, Griffin MR, Liu X, Stone WJ, Wallston KA, et al. Improving blood pressure control through provider education, provider alerts, and patient education: a cluster randomized trial. Ann Intern Med. 2006;145(3):165-175.

3. Bourbeau J, Julien M, Maltais F, Rouleau M, Beaupre A, Begin R, Renzi P, et al. Reduction of hospital utilization in patients with chronic obstructive pulmonary disease: a disease-specific self-management intervention. Arch Intern Med. 2003;163(5):585-591. 
4. Petrie KJ, Muller JT, Schirmbeck F, Donkin L, Broadbent E, Ellis CJ, Gamble G, et al. Effect of providing information about normal test results on patients' reassurance: randomised controlled trial. BMJ. 2007;334(7589):352.

5. Hiss RG, Anderson RM, Hess GE, Stepien CJ, Davis WK. Community diabetes care. A 10-year perspective. Diabetes Care. 1994;17(10):1124-1134.

6. Rich MW, Beckham V, Wittenberg C, Leven CL, Freedland KE, Carney RM. A multidisciplinary intervention to prevent the readmission of elderly patients with congestive heart failure. N Engl J Med. 1995;333(18):11901195.

7. Katon WJ, Lin EH, Von Korff M, Ciechanowski P, Ludman EJ, Young B, Peterson D, et al. Collaborative care for patients with depression and chronic illnesses. N Engl J Med. 2010;363(27):2611-2620.

8. Katayama K, Yamaguchi A, Kato M, Nakamura T, Takamatsu S, Habu D, Ito D, et al. Viewpoints on the providing of information about liver disease to patients with chronic liver disease in classes on liver disease. Kanzo. 2009;50:356-361.

9. Sorensen K, Van den Broucke S, Fullam J, Doyle G, Pelikan J, Slonska Z, Brand H. Health literacy and public health: a systematic review and integration of definitions and models. BMC Public Health. 2012;12:80.

10. Bostock S, Steptoe A. Association between low functional health literacy and mortality in older adults: longitudinal cohort study. BMJ. 2012;344:e1602. 